\title{
Verbal Control of Nonverbal Behavior ${ }^{1}$
}

\author{
DAVID BIRCH \\ University of Michigan
}

\begin{abstract}
The command, "Push the bar all the way down and hold it down" was given to 47 children 2-7 years of age. In Part 1 the command was given three times at 3-minute intervals. Results showed that all $S_{s}$ did depress the lever completely but that younger Ss gradually let up more than did

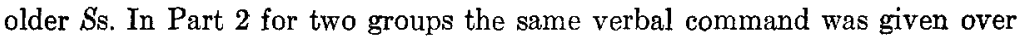
a 5-minute period either periodically at 15 -second intervals or contingent on $S$ 's letting up on the lever. For two other groups the content of the command was ascribed to the sound of a buzzer with the buzzer then used in the periodic or contingent manner. Results showed that older Ss maintained their performance under both the verbal and buzzer conditions but that younger $S$ s were able to do so under the verbal condition only. No differences were found due to periodic or contingent presentation.
\end{abstract}

Any list of the variables known to control the behavior of human beings must certainly include the spoken command. Verbal control of behavior is prevalent and powerful though not unfailing. Despite its inportance the topic of verbal control has been investigated little, (Bijou and Baer, 1966), and the available data are meager (Ayllon and Azrin, 1964). Luria (1961) in reporting work from the USSR describes changes in the effectiveness of verbal commands as the child develops from an inarticulate to an articulate organism. As the child grows he increases his capabilities for responding to external verbal commands, for ascribing the content of a verbal command to a signal and for internalizing verbal commands. The last is seen by Luria as the basic ingredient in the genesis of voluntary control in humans.

Only the external verbal control of overt nonverbal behavior will be investigated in the present experiment. An analysis of the nature of such verbal control needs to take account of two distinct sets of questions. The first set asks about the characteristics of verbal commands that determine their effectivencss for children of different agcs and the second for a

'This research was carried out under the Program-Project in the Development of Language Functions in the Center for Human Growth and Development and supported by NICH HD 01368-02. Phillip Newman collected the data and Linda Townes assisted with the analyses. 
description of the processes by which verbal control is exerted over behavior.

What makes a particular command effective for a child of a given age but ineffective for a younger child? Adequate informational and motivational inputs would both secm to be necessary. A child would be expected to perform a specific bit of behavior on command only if he understood the informational content of the command and if he were not more strongly motivated to engage in some other activity. Failures to carry out external verbal commands might also occur because of breakdowns in execution. A child might well understand and be most highly motivated by a command to jump and touch the ceiling but simply be unable to do so. The importance of the informational and executional functions for successful performance after an external verbal command showed clearly in some unpublished work from our laboratory. Following the procedures of Luria (1961), $E$ told children to press a lever down once. Young children often fail this task by pressing the lever repeatedly or by holding the lever down, as reported by Luria and replicated in our data. Attempts to account for these failures made it apparent that breakdowns could have occurred at both the informational and executional stages. The child could either have executed perfectly a misunderstood command, he could have made a faulty execution of a perfectly understood command, or he could have made a faulty execution of a misunderstood command. In the present experiment an attempt was made to minimize possible breakdowns in the informational and executional functions and to focus attention on the motivational component.

Attention to the motivational component leads directly to the second set of questions about verbal control, those pertaining to the nature of the processes that go on in the time between the reception of the command by the child and his overt performance. ${ }^{2}$ It is assumed that at any moment in time there are present in an organism tendencies to engage in a variety of incompatible activities and the activity with the strongest tendency is the one that is expressed overtly. Two processes which operate to change the strength of tendencies are also assumed to occur: (1) overt expression of a tendency acts to reduce the strength of that tendency and (2) an external verbal command functions to inerease the strength of the tendency defined by the content of the command.

Thus, if a child, engaged in one activity is commanded to engage in another, he will shift to the new activity only when that tendency becomes stronger than the tendency supporting the ongoing activity. This will occur when the command strengthens the tendency for the new

\footnotetext{
${ }^{2}$ The conceptualization that follows is adapted from a theory of motivation under
} development by John W. Atkinson and the author. 
activity to the point where it dominates that for the ongoing activity. which may be decreasing in strength because it is being expressed. The latency of the new activity, which may be described equally well as the persistence of the ongoing activity since the clock reading is the same, is the time it takes for the processes of strengthening and weakening to produce the new dominance order on the tendencies. On the other hand, if it is the ongoing activity that is called for by the verbal command no shift in behavior would be expected but possibly increased vigor or persistence of that activity. The increased strength of the tendency for the ongoing behavior would have its source in the instigation of the command.

In the present experiment verbal commands were given under both conditions; that is, where the ongoing behavior was not the same as that defined by the command and where it was. Data were sought on three major points: (1) the effectiveness of external verbal commands, both initially and with repetition, for children of different ages, (2) the persistence of the effect of external verbal commands over time as the defined activity is occurring and (3) the extent to which the instigating force of a verbal command could be ascribed to a neutral, nonverbal signal as a function of the age of the child.

\section{METHOD}

\section{Subjects}

Twenty-eight boys and nineteen girls, aged 2 years, 2 months to 7 years, served in the experiment. All Ss were enrolled in the Perry Nursery School, ${ }^{3}$ a school supported by the United Fund for the children of working mothers. The $S$ s, including six pairs of siblings, were assigned to conditions haphazardly except for a general attempt to represent the age range across the conditions. Data from $47 \mathrm{Ss}$ were collected in Part 1 of the experiment; two of these Ss failed to complete Part 2.

\section{Apparatus}

The lever depressed by $S$ was the nose of a clown's face painted on a piece of masonite approximately 1 - $\mathrm{ft}$ square. The face is painted in blue, pink, white and red and includes felt ears and eyebrows, a green glass right eye, a red glass left eye and a small speaker as a mouth. The clown's face stood upright on a grey stand in easy reach of $S$. The lever is spring loaded with a possible displacement of about $4 \frac{1}{2}$ inches and required $714 \mathrm{gm}$ for full depression.

We wish especially to thank Mrs: Elizabeth McHale; director of the Perry Nursery School for her assistance and cooperation. 
A continuous record of the extent of the lever press on a scale from 0 to 100 was obtained on a VOM-5 chart recorder set so that the paper travelled at the rate of $5 \mathrm{~mm}$ every 3 seconds. The $E$ used a hand switch to activate a second pen which served to mark the occurrence of critical events in the experiment. The chart recorder was out of the sight of $S$ and the event marking was accomplished without $S$ 's knowledge.

\section{Procedure}

The apparatus was installed in an experimental trailer, stationed in the parking lot immediately outside the school. The $E$ brought each child to the trailer individually. After minor preliminary conversation, each $S$ was scated in front of the clown's face and the following instructions were given by $E$ :

"I have some things I'd like you to do today. I will tell you what to do and after I tell you, you will do what I say. Okay?

The first thing I'm going to tell you to do is to push the bar all the way down and hold it down. When I tell you to do this, you go ahead and do it.

Push the bar all the way down and hold it down."

The last command "Push the bar all the way down and hold it down" was repeated again after 3 minutes and again after 6 minutes. At the end of 9 minutes $E$ said, "OK, you can let up on the bar now," concluding Part 1 of the experiment.

In Part 2, which followed immediately $S$ was assigned to one of four conditions, Verbal Periodic $(N=11)$, Verbal Contingent $(N=12)$, Buzzer Periodic $(N=12)$ or Buzzer Contingent $(N=12)$. To both verbal groups $E$ said, "I will tell you to push the bar all the way down and hold it down. When you hear me say this, push the bar all the way down and hold it down." For the Verbal Periodic group the command was repeated every 15 seconds; for the Verbal Contingent group the same command was repeated once whenever $S$ began to let up on the lever. Similarly for the two buzzer groups, $E$ sounded the buzzer a few times and said, "Now this time the buzzer is going to tell you to push the bar all the way down and hold it down. When you hear the buzzer, push the bar down and hold it down." The buzzer was sounded every 15 seconds for the Buzzer Periodic group and whenever $S$ let up on the lever for the Buzzer Contingent group. At the end of five minutes $E$ told all $S$ s, "Now you can let up on the bar."

\section{RESULTS}

The extent of the depression of the lever was recorded continuously for each $S$. In order to obtain data suitable for statistical analysis the 
continuous records were changed into discrete form by reading off the extent of the depression at each successive 3 -second point. Mean values for successive blocks of five readings were calculated to give the basic unit of analysis.

The first time the Ss were given the external verbal command to "Push the bar all the way down and hold it down" all depressed the lever to its full extent within the subsequent three minutes. The speed with which this shift in activity occurred is significantly and positively rclated to

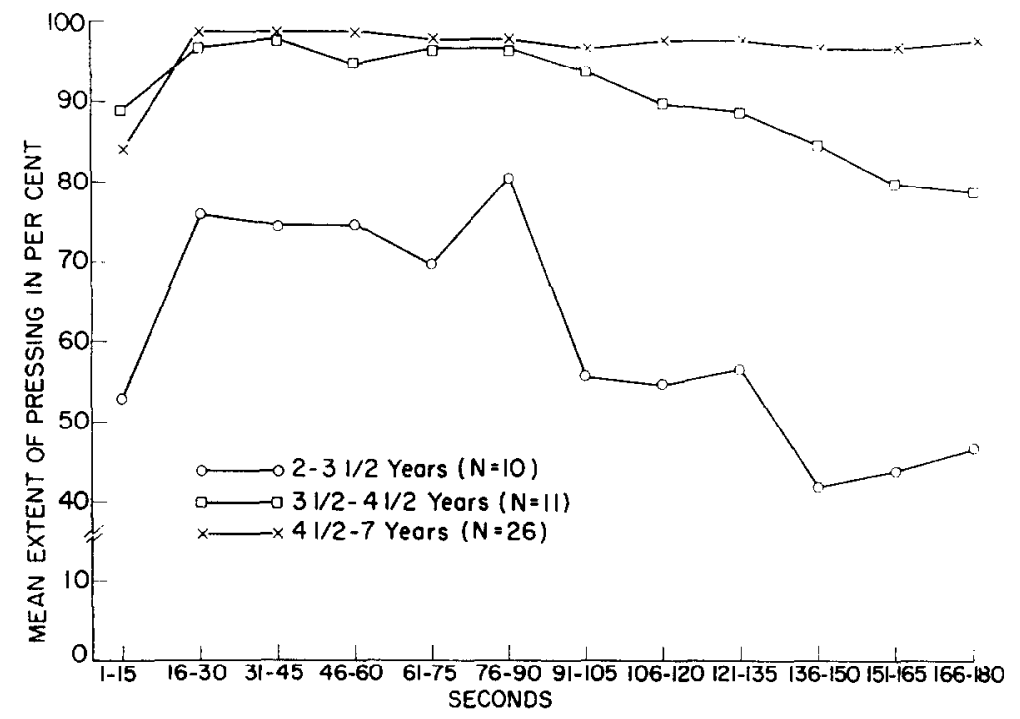

FIg. 1. Extent of lever pressing over 3 minutes after a single verbal command for children of different age levels.

age. The product moment correlation between age and the time it took to attain the first full lever depression is significant $(r=-.32, d f=45$, $p<.05)$ as is the correlation between age and mean percentage of lever depression during the first 15-second period after the command $(r=.42$, $d f=45, p<.01$ ).

The effectiveness of the first verbal command for Ss of different ages was examined further by tracking the percentage of lever depression over the whole 3-minute period following the command. Ss were grouped into three age levels, $3 \frac{1}{2}$ years and younger, over $3 \frac{1}{2}$ up to and including $41 / 2$ and older than $4 \frac{1}{2}$. The results are pictured in Fig. 1.

It is easily seen that both the overall percentage of depression and the decline in percentage depression over time are related to age level. The group with the oldest Ss shows the highest average depression $(97 \%)$ and maintains the lever press throughout the whole 3 -minute period while the 
groups with the intermediate aged and youngest $S$ s average less overall depression (91\% and $61 \%$, respectively) and exhibit a decline in their extent of pressing over time.

Analysis of variance on these data shows both main effects and the interaction to be significant beyond the .001 level; for Age Lcvels $F(2,44)=27.87$, for Time $F(11,484)=6.15$ and for the Age Level $\times$ Time interaction $F(22,484)=3.15$. Examination of the data also shows that 3 of the $10 \mathrm{Ss}$ in the youngest group, 1 of the $11 \mathrm{Ss}$ in the intermediate aged group and none of the $26 \mathrm{Ss}$ in the oldest group have stopped pressing altogether by the end of 3 minutes. On the other hand 1 of the youngest $S \mathrm{~s}, 7$ of the intermediate aged and 24 of the oldest $S \mathrm{~s}$ are still depressing the lever beyond the $95 \%$ point at the end of that time period.

TABLE 1

Mean Percentage Lever Depression of the Three Age Groups for the 3 Minutes after Each of the Three Commands to "Pheiss the Lever Down and Hold It Down"

\begin{tabular}{|c|c|c|c|c|c|c|c|c|c|}
\hline \multirow[b]{3}{*}{ Command } & \multicolumn{3}{|c|}{$\begin{array}{c}\leq 31 / 2 \text { Years } \\
(N=10)\end{array}$} & \multicolumn{3}{|c|}{$\begin{array}{c}31 / 2-41 / 2 \text { Years } \\
(N=11)\end{array}$} & \multicolumn{3}{|c|}{$\begin{array}{c}>41 / 2 \text { Years } \\
(N=26)\end{array}$} \\
\hline & \multicolumn{6}{|c|}{ Minute } & & & \\
\hline & 1 & 2 & 3 & 1 & 2 & 3 & 1 & 2 & 3 \\
\hline 1 & 70 & 65 & 46 & 95 & 94 & 83 & 96 & 98 & 98 \\
\hline 2 & 59 & 45 & 48 & 91 & 76 & 73 & 98 & 96 & 96 \\
\hline 3 & 56 & 39 & 37 & 83 & 73 & 70 & 94 & 93 & 86 \\
\hline Meau & 61 & 49 & 44 & 90 & 81 & 76 & 96 & 96 & 93 \\
\hline
\end{tabular}

The lever depression scores of these same three age groups for all three commands are shown in Table 1 . The entrics are mean percentages of lever depression for each of the 3 minutes after each of the three commands. These data, evaluated by analysis of variance, replicate the results found for the first command alone. That is, there is greater overall lever depression for older $S \mathrm{~s}, F(2,44)=23.30, p<.001$ for the Age Groups main effect, a significant decline in lever depression over time after a command, $F(2,88)=14.99, p<.001$ for this main effect, and a faster rate of decline in lever depression over time for younger $S$ s as seen in the Age Level $\times$ Time interaction, $F(4,88)=5.36, p<.001$. In addition, as may be discerned from Table 1, each of the last two commands is less effective than its predecessor. For the Commands. main effect $F(2,88)=5.56, p<.01$. No interactions involving Commands approached significance. 
In the second part of the experiment sis were assigned to one of four experimental conditions defined by the combination of the two types of command (verbal or buzzer) and the two modes of presentation (periodic or contingent). Two Age Groups were also formed, the younger group included those Ss $4 \frac{1}{2}$ years and less and the older, those more than $4 \frac{1}{2}$ years. A preliminary examination of the data, performed because of the disproportionate number of $S_{s}$ in the cells, showed the mode of presentation to be irrelevant. Therefore, Ss were pooled over the periodic and contingent conditions for subsequent analysis of variance. Preliminary

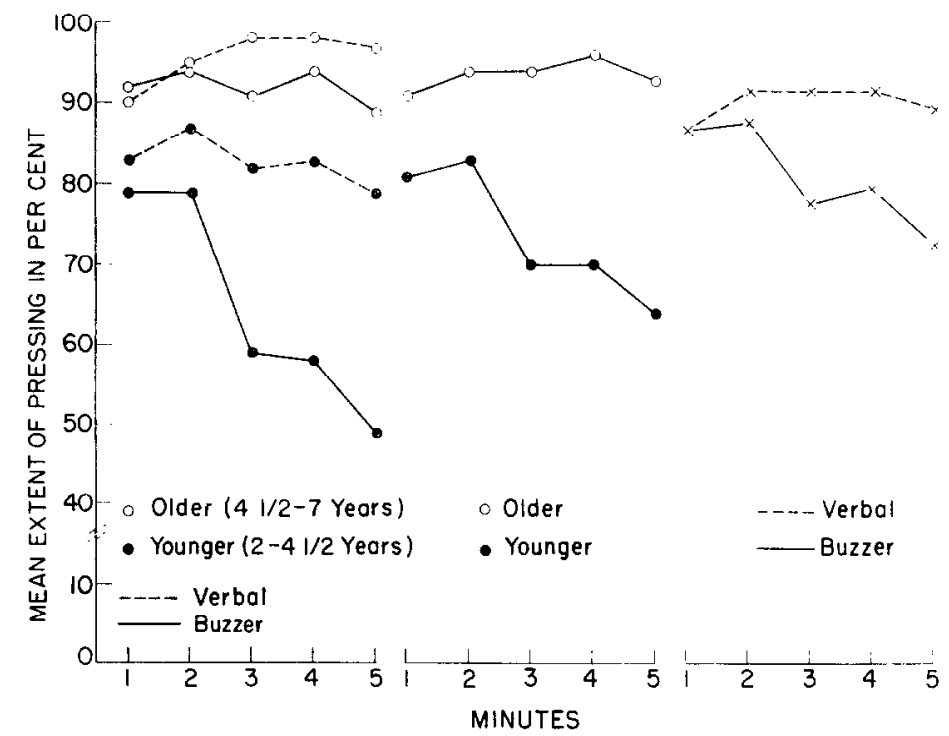

Fig. 2. Extent of lever pressing over 5 minutes of repeated commands, verbal or buzzer, for younger and older children.

examination also showed that the nonsystematic assignment of Ss to the verbal and buzzer groups did not equate these groups for performance on Part 1 of the experiment. This was due partly to two $S$ s who did not complete the second part of the experiment and partly to three $S_{s}$ in the buzzer group who were the poorest performers in the first part of the experiment. By eliminating these five $S$ s and matching the remaining $S$ s in terms of their overall performance on Part 1, a final set of data, arranged in a three-dimensional factorial design made up of Age Group, Type of Command and the correlated dimension of Time, was arrived at. Two more Ss. were lost in carrying out the matching leaving a total $N=40$. The two younger groups. (verbal or buzzer) each have. $N=8$ and the two older groups each have $N=12$. Analysis of variance on the 
data for these Ss from Part 1 of the experiment yielded $F<1$ for all effects involving the verbal and buzzer types of command to be used in Part 2 indicating the matching was satisfactory.

Plots of the data for Part 2 of the experiment are displayed in Fig. 2. The first panel shows the percentage lever depression for the four groups. The difference in level of depression as related to age is easily observable in the plot but most apparent is the relatively stable level of pressing for all groups except the Younger-Buzzer group which reduces its responding markedly during the 5-minute period. This effect shows up in the other two panels where the overall effects of Age Level and Type of Command are plotted as a function of time. Analysis of variance indicates significance for the Age Level, $F(1,36)=7.15, p<.05$, and Time, $F(4,144)=2.78, p<.05$, main effects and for the Time $\times$ Type of Command, $F(4,144)=3.81, p<.01$, and the Time $\times$ Age Level, $F(4,144)=$ $5.27, p<.001$, interactions. The Type of Command main effect and the two interactions failed to approach significance. A subscquent analysis of variance on the data for the first minute only yielded nonsignificance.

\section{DISCUSSION}

The present experiment was designed to yield data on two topics; (1) the characteristics of verbal commands that determine their effectiveness for children of different ages and (2) the processes by which verbal control is exerted over behavior. The simple command, "Push the bar all the way down and hold it down" was used. All Ss responded adequately to this command giving evidence that they understood the command and were able to perform the required motor responses. Following the first command no $S$ failed to depress the lever to its full extent and all $S$ s continued to press for an appreciable interval. Only four $S$ s, all in the youngest group, had a first press duration of less than a minute, their durations being $9,9,24$ and 45 seconds. With this assurrance that the informational and executional components of successful verbal control were satisfactory, it was possible to focus attention on the motivational component and to investigate the effect of variations in presentation of the command.

The findings on characteristics determining the effectiveness of commands for $S_{s}$ across the age range 2-7 years were as follows: (1) The verbal command given singly differs in both initial and persisting effectiveness as a function of age with the older children performing more proficiently than the younger. (2) Irrespective of the age of $S$ the verbal command loses effectiveness with repetition at 3-min. intervals but not at shorter intervals. (3) The initial transfer of the content of the verbal command to a buzzer is satisfactory for the whole age range but the 
buzzer loses effectiveness with repetition for the younger Ss. (4) No effect of periodic as opposed to contingent presentation was discernable. These relations between the effectiveness of verbal control and age agree with the findings of Luria (1961) on the same topic and emphasize again the importance of the years about three to five in the development of the child's verbal skills. Other investigators dealing with different tasks (e.g., Kuenne, 1946; Weir and Stevenson, 1959; Kendler and Kendler, 1962; and White, 1965) point to crucial changes occurring in this same period, changes which also are likely to be related to the development of the verbal system of the child.

In proposing a description of the processes going on in verbal control it was hypothesized that an external verbal command functions to increase the strength of the tendency to engage in the activity defined by the command and that expressing a tendency in overt activity acts to reduce the strength of that tendency. Both hypotheses are supported by the results of the present experiment under the assumption that the strength of the tendency to hold the lever down is reflected directly in the depression of the lever. In Fig. 1 these effects are illustrated by the marked increase in the depression of the lever after the initial command and the subsequent decline, at least for the two groups of younger $S \mathrm{~s}$, as the pressing continues. As shown in Table 1 this same pattern of rise and fall in strength of tendency follows upon each of the thrce verbal commands administered in Part 1. Examination of the same data for individuals indicates that the graphs are smoothed considerably by averaging into groups but that individual results are not misrepresented. That is, individuals, like groups, tend to show a gradual weakening of the tendency to hold down the lever after performing the initial press.

A question arises with regard to the older $S \mathrm{~s}$, however, as to why the tendency remains at high strength throughout the period after an external verbal command even though the activity is occurring and should be reducing the strength of the supporting tendency. This result holds consistently for the older Ss throughout the experiment. The answer might be that the tendency remains strong because it continues to be instigated. This could occur in Part 1 if the older Ss provided themselves with internally originated verbal commands (i.e., if from time to time these $S_{\mathrm{s}}$ in effect said to themselves, "Push the bar all the way down and hold it down"). They might also do this in Part 2 though it would not be necessary since $E$ presents repeated external commands, either verbally or by buzzer.

Applying this speculation to the data for the younger $S$ s leads to the conclusion that their tendency to hold the lever down declines in Part 1 because they fail to provide themselves with the necessary self-instruc- 
tions. In Part 2 when $E$ gives repeated verbal commands they perform as well as the older $S s$, but when the buzzer is employed their performance deteriorates, presumably because the buzzer loses its instigating power for them. The difference in results for these two groups of younger $S$ s is important in another respect. The fact that repeated administrations of the verbal command maintains the lever pressing activity over a 5 -minute period is convincing evidence that the decline in pressing during Part 1 of the experiment and in the buzzer condition of Part 2 is not due simply to a lack of muscular strength in these younger $S$ s. As the data show they are fully capable of continuing to depress the lever over this extended period, but they do not do so unless repeatedly exposed to the verbal command. Thus, the findings are consistent with the hypotheses that verbal commands function to increase the strength of tendencies, that tendencies are reduced in strength when they are expressed in overt action and that repeated application of a verbal command is necessary in order to maintain the strength of a tendency that is bcing expressed in action.

\section{REFERENCES}

Ayllon, T., AND AzRin, N. H. Reinforcement and instructions with mental patients. J. exp. anal. Behav., 1964, 7, 327-331.

BiJou, S. W., AND BaER, D. M. Operant methods in child behavior and development. In W. K. Honig (Ed.) Operant behavior: areas of research and application. New York: Appleton-Century-Crofts, 1966.

KendleR, H. H., and Kendler, Tracy S. Vertical and horizontal processes in problem solving, Psychol. Rev., 1962, 69, 1-16.

Kuenne, Margaret R. Experimental investigation of the relation of language to transposition behavior in young children. J. exp. Psychol, 1946, 36, 471-490.

LURIA, A. R. The role of speech in the regulation of normal and abnormal behavior. New York: Liveright, 1961.

Weir, M. W. ANd Stevenson, H. W. The effect of verbalization in children's learning as a function of chronological age. Child Develpm., 1959, 30, 143-149.

WHIte, S. H. Evidence for a hierarchical arrangement of learning processes. In L. P. Lipsitt and C. C. Spiker (Eds.), Advances in child development and behavior. Vol. 2. New York: Academic Press, 1965. 